\title{
SQUARE CYLINDER UNDER DIFFERENT TURBULENT INTENSITY CONDITIONS BY MEANS OF SMALL-SCALE TURBULENCE
}

\author{
ANTONIO J. ÁLVAREZ ${ }^{1}$, FÉLIX NIETO ${ }^{1}$, KENNY C. S. KWOK ${ }^{2}$ \& SANTIAGO HERNÁNDEZ ${ }^{1}$ \\ ${ }^{1}$ Structural Mechanics Research Group, University of La Coruña, Spain \\ ${ }^{2}$ School of Civil Engineering, The University of Sydney, Australia
}

\begin{abstract}
The phenomenon of turbulence is present in almost every type of flow in practical applications. Depending on its level of intensity and length scale, it can modify both the aerodynamic and aeroelastic performance of a body under flow action. In wind tunnel tests, the desired turbulence level is achieved by placing obstacles, spires, grids and extra roughness generators upwind the tested model. On the other hand, when trying to reproduce turbulence effects by means of a computational fluid dynamics (CFD) approach, two options have usually been considered: synthetic turbulence generation and the reproduction of velocity and pressure fluctuations recorded from previous simulations or wind tunnel tests. Another option, whose feasibility in CFD applications is addressed in this work by means of a 2D URANS (unsteady Reynolds averaged Navier-Stokes) consists of placing a rod upstream of the studied body, near the stagnation line. This approach is based on the generation of small scale turbulence upstream of the studied body, so that the turbulent wake generated by an upwind rod impinges on the body located downwind. In the present study, by means of 2D URANS simulations, the smooth flow over a circular cylinder (the upwind rod) is studied focusing on its wake turbulence characteristics. Furthermore, the aerodynamic performance of a square cylinder, first under smooth flow, and later immersed in the turbulent wake of the upstream rod, are analysed. A substantial effort has been devoted in the verification studies of the numerical models. It has been found that the adopted numerical approach is able to reproduce the turbulent characteristics of the rod wake and assess the impact of the turbulent flow on a square cylinder, providing a promising agreement with experimental data.

Keywords: 2D URANS, rod-generated turbulence, small scale turbulence (SST).
\end{abstract}

\section{INTRODUCTION}

Structures in the built environment are immersed in the atmospheric boundary layer, which is turbulent in nature. Therefore, the assessment of the aerodynamic and aeroelastic responses of structures encountering turbulent wind is of utmost importance to guarantee its safety and efficient performance.

The standard approach to study the effect of turbulence on a body is to conduct wind tunnel tests. In boundary layer wind tunnels, obstacles such as spires and roughness elements placed on the lower surface, are used to obtain the desired profile of mean velocity and turbulent intensity, representative of the turbulent atmospheric boundary layer. Alternatively, a grid placed upstream of the studied model may be used to generate a turbulent flow with the desired uniform mean speed and turbulent characteristics, without intending to replicate a boundary layer profile. Gartshore [1], proposed a different approach to generate small scale free stream turbulence by placing a rod along the stagnation line upstream of the studied body, to produce the major effects of free stream turbulence with the same turbulence intensity. This method, has been successfully applied, for instance, in Kwok and Melbourne [2] and Kwok [3]; and more recently in Lander et al. [4], to study the shear layer development of bluff bodies in a turbulent flow by means of Time Resolved Particle Image Velocimetry (TR-PIV). 
Addressing free stream turbulence in CFD simulations represents a challenge due to the intrinsic unsteadiness, three dimensionality, broad range of scales and randomness in the incoming flow. According to Patruno and Ricci [5], there are two different methodologies to tackle this problem by adopting scale-resolving turbulence models such as Large Eddy Simulation (LES): one based on extracting the turbulent fluctuations from an auxiliary simulation or the recycling of the velocity in a plane within the simulation itself; and the generation of synthetic random fields. Both methods are complex and computational expensive, requiring a careful preparation [6], [7]. On the other hand, the use of URANS models to address free stream turbulence has been mainly confined to urban applications [8][10] due to its sufficient reliability for the intended applications and the much higher computational cost of scale-resolving alternative approaches.

This piece of research aims at assessing the feasibility of adopting a 2D URANS approach to study, at least qualitatively, the aerodynamic response of bluff bodies under free stream turbulent flow. To this end, the small-scale rod-generated turbulent flow approach proposed by Gartshore [1] for wind tunnel testing has been adopted in the computational simulations reported in this paper. A static square prism under smooth and 3.3\% turbulent intensity (TI) free stream flows has been studied, comparing the numerical results with experimental data presented in Gartshore [1] and Lander et al. [4], as well as additional experimental data in the open literature. A good agreement has been found for important outputs such as the drag coefficient, pressure coefficient distributions and time-averaged streamlines. Hence, this preliminary study has shown promising results, suggesting undertaking further studies on this 2D URANS approach that decreases the computational burden of numerically studying turbulence effects while provides reliable results for wind engineering applications.

\section{FORMULATION}

\subsection{Governing equations}

The time averaging of the Navier-Stokes equations in a conservative form yields the URANS eqns [10]:

$$
\begin{gathered}
\frac{\partial U_{i}}{\partial x_{i}}=0,- \\
\rho \frac{\partial U_{i}}{\partial t}+\rho U_{j} \frac{\partial U_{i}}{\partial x_{j}}=-\frac{\partial P}{\partial x_{i}}+\frac{\partial}{\partial x_{j}}\left(2 \mu S_{i j}-\rho \overline{u_{i}{ }^{\prime} u_{j}{ }^{\prime}}\right)
\end{gathered}
$$

where $U_{i}$ is the mean velocity vector, $x_{i}$ is the position vector, $\rho$ is the fluid density, $t$ is the time, $P$ is the mean pressure, $\mu$ is the fluid viscosity, $S_{i j}$ is the mean strain-rate tensor and $u_{i}^{\prime}$ is the fluctuating velocity.

The term $-\overline{u_{\imath}^{\prime} u_{\jmath}^{\prime}}$ is the so-called specific Reynolds stress tensor $\left(\tau_{i j}\right)$, calculated by means of the Boussinesq assumption as:

$$
\tau_{i j}=2 v_{T} S_{i j}-\frac{2}{3} k \delta_{i j}
$$

where $v_{t}$ is the kinematic eddy viscosity and $k$ is the kinetic energy per unit mass of the turbulent fluctuation.

The different closure equations added to the previous ones, define the type of URANS model obtained. In this piece of research, the model selected is the $k-\omega$ SST, for 
incompressible flow, implemented in the open source software OpenFOAM, whose formulation was proposed by Menter and Esch [11].

\subsection{Force coefficients, Strouhal number, pressure coefficient and base pressure coefficient}

The time-dependent force coefficients $\left(\operatorname{drag}\left(C_{d}\right)\right.$, lift $\left(C_{l}\right)$ and moment $\left.\left(C_{m}\right)\right)$ along with the Strouhal $(S t)$ number, also referred to as integral parameters, are calculated according to eqn (4):

$$
C_{d}=\frac{F_{D}}{\frac{1}{2} \rho U^{2} D_{s}}, C_{l}=\frac{F_{L}}{\frac{1}{2} \rho U^{2} D_{s}}, C_{m}=\frac{M}{\frac{1}{2} \rho U^{2} D_{s}^{2}}, S t=\frac{f D_{s}}{U}
$$

where $D_{S}$ stands for the side of the square cylinder (see Fig. 1), $\rho$ is the air density, $U$ is the free-stream velocity, $f$ is the dominant frequency of the lift coefficient, and $F_{D}, F_{L}$ and $M$ are the drag and lift forces and moment per unit of length respectively, which were calculated as the spanwise averaging of the integration of the pressure and viscous forces along the twinbox surfaces. The sign convention of the force coefficient is depicted in Fig. 1.

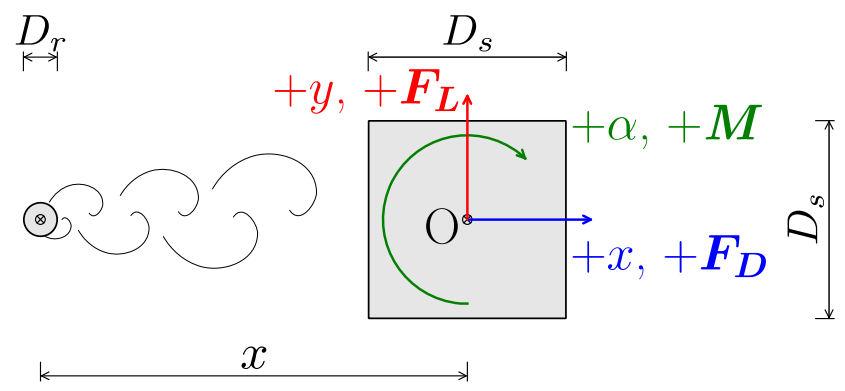

Figure 1: Sign convention $\left(D_{s}\right.$ stands for the square cylinder side length and $D_{r}$ for the rod diameter).

In the following, the time-averaged force coefficients values will be referred as $\overline{C_{k}}$ and their standard deviations as $\widetilde{C_{k}}(k=d, l, m)$.

The mean pressure coefficient $\left(\overline{C_{p}}\right)$ and its standard deviation $\left(\widetilde{C_{p}}\right)$ are calculated as shown by eqn (5):

$$
\overline{C_{p}}=\frac{\bar{p}}{\frac{1}{2} \rho U^{2}}, \widetilde{C_{p}}=\frac{\tilde{p}}{\frac{1}{2} \rho U^{2}},
$$

The base pressure coefficient $\left(C_{p b}\right)$ is calculated as indicated in eqn (6). The integral of the pressures is done over the complete leeward face of the square cylinder.

$$
C_{p b}=\frac{\int_{o}^{D_{s}} \bar{p}(s) d s}{\frac{1}{2} \rho U^{2} D_{s}} .
$$




\subsection{Turbulence intensity}

According to Simiu and Scanlan [12], the longitudinal turbulence intensity of a wind flow $\left(I t_{u}\right)$ is:

$$
I t_{u}=\frac{\widetilde{U_{x}}}{U},
$$

with $\widetilde{U_{x}}$ being the standard deviation of the longitudinal component of the wind. As the wind flow is obtained by means of URANS simulations, this value would only refer to the variation of the mean velocity, thereby missing the contribution of the fluctuating component. The fluctuating components are the ones yielding the specific Reynolds stress tensor, and according to Lander et al. [4] the turbulence intensity due to the fluctuating components of the longitudinal component of the velocity $\left(I t_{R}\right)$ is:

$$
I t_{R}=\frac{\sqrt{\overline{u_{x}^{\prime} u_{x}^{\prime}}}}{U}=\frac{\sqrt{\tau_{x x}}}{U},
$$

where $\tau_{x x}$ is the longitudinal component of the specific Reynolds stress tensor. Hence the total turbulence intensity $(I t)$ is the summation of both contributions:

$$
I t=I t_{u}+I t_{R}
$$

\section{MODELLING AND COMPUTATIONAL APPROACH}

Three types of simulations have been performed in this study: simulations of the isolated rod, simulations of the isolated square cylinder and simulations of the square cylinder in the wake of the rod. The side of the square cylinder $\left(D_{S}\right)$ is twelve times the diameter of the rod $\left(D_{r}=D_{s} / 12\right)$, and all the simulations have been conducted at a Reynolds number, calculated with respect $D_{s}, \operatorname{Re}=3.84 \times 10^{4}$, the same used in the experiments conducted by Gartshore [1] that are later used for validation. These 2D URANS simulations were conducted by means of the $k-\omega$ SST turbulence model implemented in the CFD software OpenFOAM. The diffusive terms are computed using a second-order differential scheme, while the convective terms use the linear upwind differential scheme. The advancement in time is accomplish by a first order implicit scheme and the pressure velocity coupling is resolved by the PIMPLE algorithm.

The overall fluid domain dimensions, for the different types of simulations, is depicted in Fig. 2(a), and its dimensions are presented in Table 1.

For the space discretisation, a non-conformal structured quadrangular mesh is used. The fluid domain has been subdivided in five different zones (see Fig. 2(b)). In each boundary between zones, the number of elements is halved from the zone with a lower identifier. In all the simulations, the mean $y^{+}$of the rod is always below 2.5 , while this value is always below 1.2 for the square cylinder. In both cases the $y^{+}$value has been calculated considering the total height of the first element of the boundary layer.

At the inlet, Neumann conditions were imposed for the pressure, while Dirichlet conditions were applied to the velocity, the specific dissipation rate and the turbulent kinetic energy. The last two values have been calculated considering an incoming turbulence intensity of $1.0 \%$ and a length scale of $0.1 D_{s}$. In the case of the outlet boundary, Neumann conditions have been considered for the velocity, the specific dissipation rate and the turbulent kinetic energy fields, while Dirichlet conditions were applied to the pressure. For 


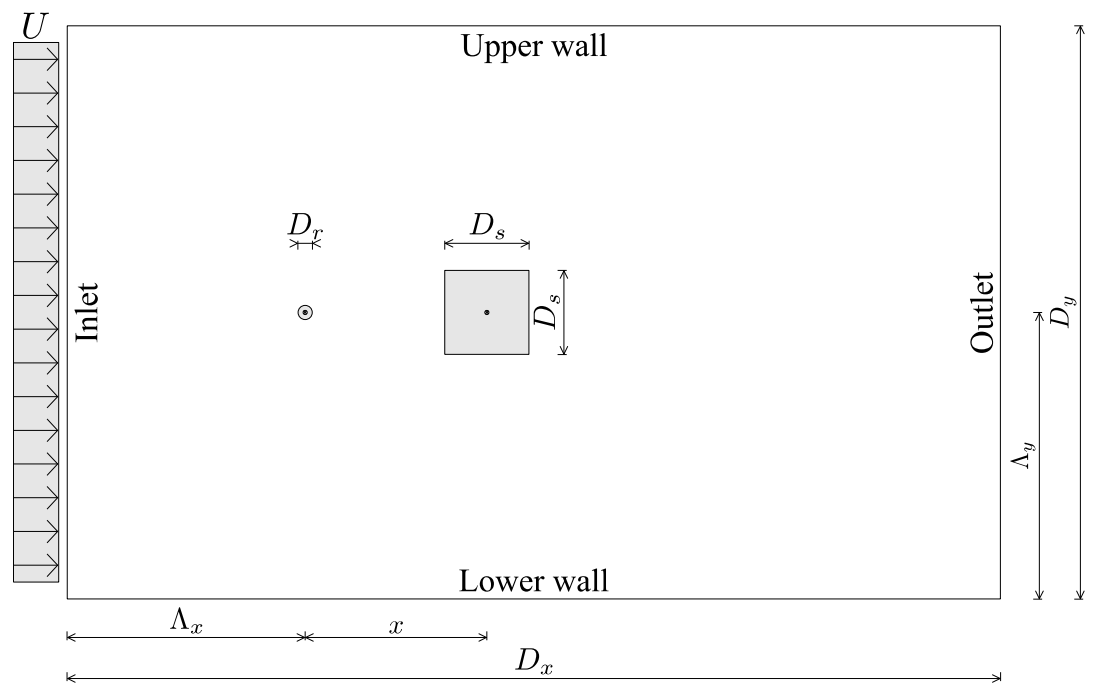

(a)

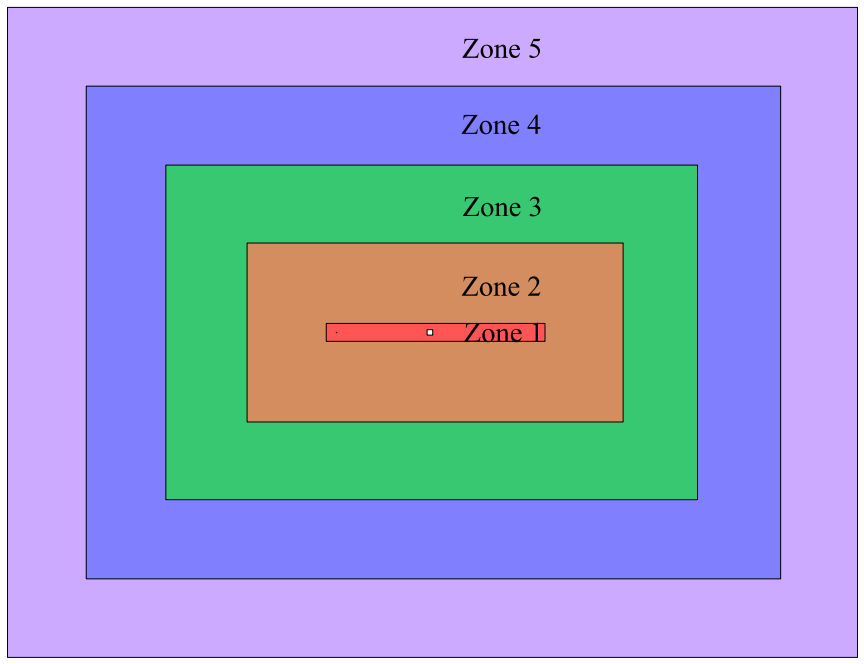

(b)

Figure 2: Fluid domain definition. (a) Overall fluid domain (not to scale); (b) Different zones in which the mesh is subdivided.

Table 1: Overall fluid domain dimensions. $D_{s}$ is the side of the square cylinder, $\Lambda x$ is the distance from the centre of the rod to the inlet boundary, $\Lambda \mathrm{y}$ is the distance from the centre of the square cylinder to both the upper and lower walls, $D x$ and $D y$ are respectively the total width and height of the fluid domain.

\begin{tabular}{cccc}
\hline$\Lambda x$ & $\Lambda y$ & $D x$ & $D y$ \\
\hline $60 D_{s}$ & $60 D_{s}$ & $160.5 D_{s}+x$ & $120 D_{s}$ \\
\hline
\end{tabular}


the upper and lower boundaries of the fluid domain a slip wall boundary condition was selected. In the deck walls, no penetration and no-slip boundary conditions were applied.

Verification studies aiming at identifying the finite volume grids insensitive to the spatial discretization have been conducted for the isolated rod and square cylinder in smooth flow. For the rod, three different grids were considered: a coarse mesh comprising 336,176 cells, a medium mesh comprising 507,984 cells and a fine mesh with 715,888 cells. It was found that the medium mesh provided results that were independent of the level of spatial discretization. For the isolated square prism, a similar study was carried out, also considering a coarse grid with 316,976 cells, a medium grid comprising 478,224 cells and a fine grid of 672,112 cells. In this case, the medium mesh grid also provided results that were insensitive to the spatial discretization level. The characteristics of the medium meshes were retained to generate the mesh combining the rod and the square prism, resulting in a 665,284 cells non-conformal structured hexahedral grid.

\section{TURBULENCE GENERATION}

Turbulence is generated by means of a circular rod placed upwind of the square cylinder, reproducing the arrangement in the experiments conducted by Gartshore [1] and Lander et al. [4]. Therefore the turbulent flow is due to the wake shed by the rod, which impinges upon the square cylinder. The turbulence intensity decays with the distance from the rod, as shown in Fig. 3, where the turbulence intensity obtained in our simulation along the centre line of the domain, downstream of the isolated rod, is compared with the data provided by Gartshore [1]. It is observed that the numerical results follow the trend of the experimental values and they present a reasonable agreement with the reported experimental values.

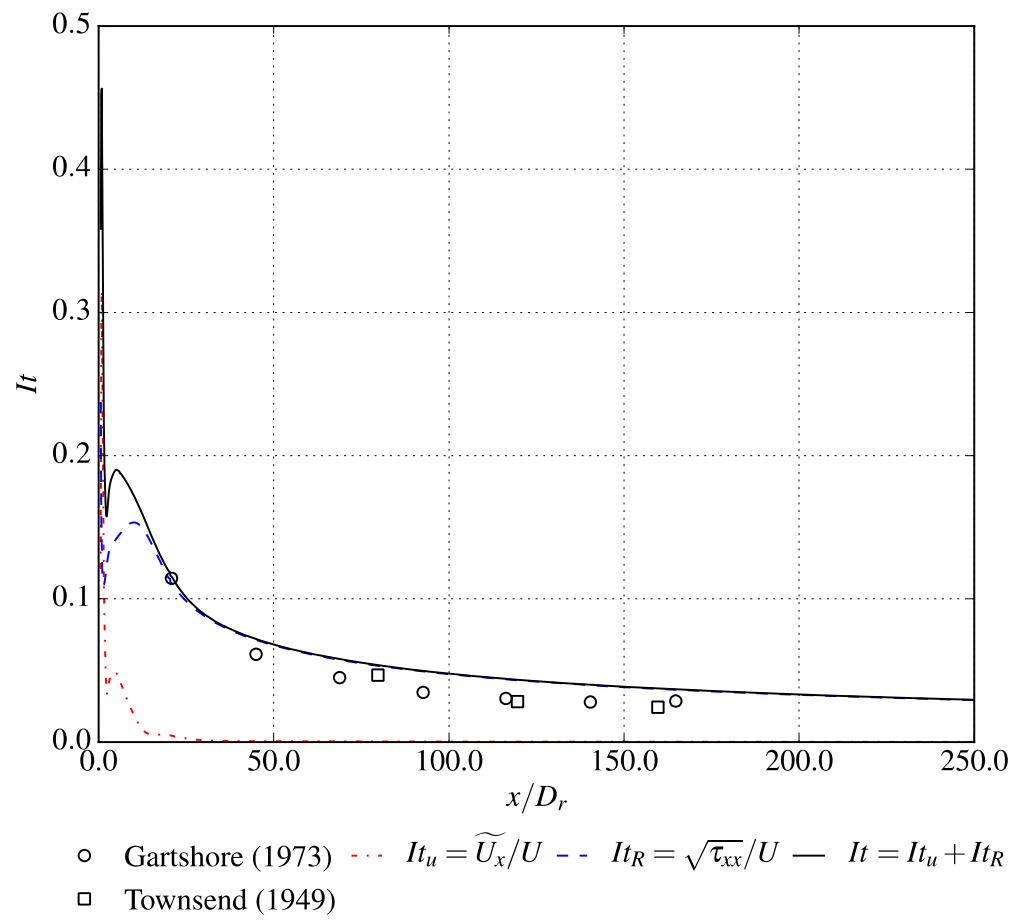

Figure 3: Longitudinal turbulence intensity in the wake of the circular rod. 


\section{RESULTS}

It is recalled that the results reported next for the square cylinder in smooth flow are obtained by means of a 2D URANS simulation comprising only the square cylinder. For the case considering a nominal turbulent intensity of 3.3\%, the computational model comprises the rod upstream of the square cylinder, separated a distance between centres $x=212.37 D_{r}$, as depicted in Fig. 1.

Fig. 4 presents the turbulence intensity profile in the wake of the rod along the stagnation line of and in the presence of the square cylinder. It is observed that the turbulence intensity diminishes as the distance from the rod increases, following the same trend as the one depicted in Fig. 3. The turbulent intensity starts to increase again in the vicinity of the square prism, reaching a maximum near the surface of the windward face.

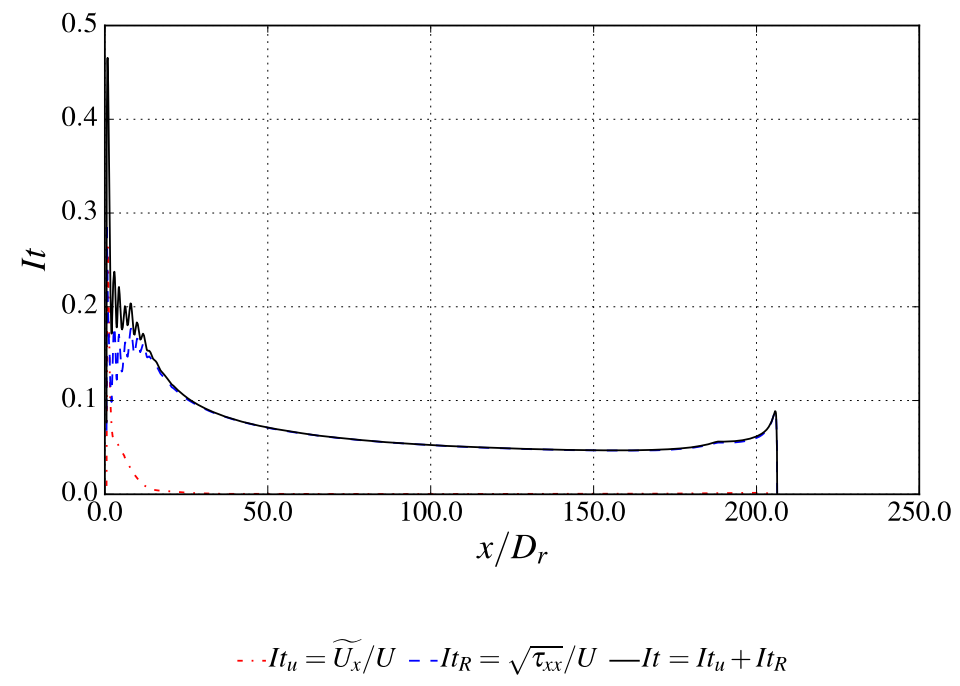

Figure 4: Longitudinal turbulence intensity in the gap between the rod and the square cylinder.

In Table 2, the force coefficients, Strouhal number and base pressure coefficient obtained for the square cylinder by the numerical simulations conducted in smooth flow are compared with available experimental results in the literature. Meanwhile in Table 3, the results for the same parameters are presented for the case considering a nominal turbulence intensity of $3.3 \%$.

Table 2: Integral parameters and base pressure coefficient for the simulation in smooth flow. (PS refers to present study).

\begin{tabular}{ccccccccccc}
\hline & $\mathrm{Re}$ & $I t \%$ & $\overline{C_{d}}$ & $\overline{C_{l}}$ & $\overline{C_{m}}$ & $\widetilde{C_{d}}$ & $\widetilde{C_{l}}$ & $\widetilde{C_{m}}$ & $S t$ & $C_{p b}$ \\
\hline PS & $3.84 \times 10^{4}$ & 0.0 & 2.06 & 0.02 & -0.01 & 0.35 & 1.35 & 0.11 & 0.12 & -1.27 \\
{$[13]$} & & 0.0 & & & & & & & & -1.31 \\
{$[14]$} & $3.7 \times 10^{4}$ & 0.2 & 2.06 & -0.02 & & & 1.02 & & 0.12 & -1.49 \\
{$[1]$} & $3.84 \times 10^{4}$ & 0.6 & 2.20 & & & & & & & -1.45 \\
{$[4]$} & $5.0 \times 10^{4}$ & 1.0 & 2.35 & & & 0.22 & 1.14 & & 0.13 & -1.51 \\
\hline
\end{tabular}


Table 3: Integral parameters and base pressure coefficient for the simulation under a total turbulence intensity (It) of 3.3\%. (PS refers to present study).

\begin{tabular}{ccccccccccc}
\hline & $\mathrm{Re}$ & $I t \%$ & $\overline{C_{d}}$ & $\overline{C_{l}}$ & $\overline{C_{m}}$ & $\widetilde{C_{d}}$ & $\widetilde{C_{l}}$ & $\widetilde{C_{m}}$ & $S t$ & $C_{p b}$ \\
\hline PS & $3.84 \times 10^{4}$ & 3.3 & 1.92 & -0.01 & 0.00 & 0.14 & 1.49 & 0.12 & 0.13 & -1.26 \\
{$[1]$} & $3.84 \times 10^{4}$ & 3.3 & 1.84 & & & & & & & -1.18 \\
{$[4]$} & $5.0 \times 10^{4}$ & 6.5 & 1.68 & & & 0.15 & 1.10 & & 0.14 & -1.22 \\
\hline
\end{tabular}

Evidently, there are fairly good agreements between the numerical and experimental results, both in smooth and turbulent flows. Moreover, the drag coefficient mean value decreases as the level of turbulence intensity increases. On the other hand, the Strouhal number is slightly increased with an increase in turbulence intensity, for the experimental values.

In Fig. 5, the mean and fluctuating pressure coefficient distributions for smooth and 3.3\% free stream turbulent flow are presented and compared with available experimental data.

The mean pressure coefficient distribution in smooth flow obtained by means of 2D URANS simulations agrees remarkably well with the experimental data in Carassale et al. [14]. For the numerical simulation considering a 3.3\% turbulent intensity, a decrease in the pressure distribution on the windward face with respect to the smooth flow is obtained, which agrees with the trend reported in Lander et al. [4] for increasing turbulence levels. In the simulation, the mean pressure distribution on both side faces is relatively insensitive to the turbulence level, as in Lander et al. [4]. On the contrary, the decrease in the suction acting on the leeward face of the cylinder, identified experimentally in Lander et al. [4], is not evident in the numerical simulations, which generated a similar mean pressure distribution for both smooth and 3.3\% turbulent flows. This circumstance will be further commented upon when referring to the mean streamlines plots.

With respect to the fluctuating component of the pressure coefficient, the numerical simulations not only reproduce the trends of the experimental values in Lander et al. [4], but are in a good agreement with the experimental data, remarkably in the reduction of the fluctuating pressure coefficient along the leeward face of the square cylinder with the increase in the free stream turbulence.

Finally, in Fig. 6, the time-averaged streamlines for smooth flow and 3.3\% free stream turbulence obtained by 2D URANS are presented and compared with the experimental data for $1 \%$ and $6.5 \%$ free stream turbulence in Lander et al. [4]. The agreement between the experimental and numerical mean streamlines for nominal smooth flow may be highlighted. It has been possible to reproduce the size of the main vortex located in both side faces of the square cylinder, as well as the mean base-region size in its wake. On the other hand, when comparing the cases with higher free stream turbulence, the main vortices on both side faces of the square cylinder obtained by the 2D URANS simulation remain very similar to the ones in smooth flow, although a reduction in length in the recirculation region is observed due to the increase in curvature and the reattachment of the shear layer close to the leeward corners. This is qualitatively consistent with experimental evidence in Lander et al. [4]. However, the length of the base region for the $3.3 \%$ turbulent intensity case has not increased, as it should be expected.

These discrepancies may be explained by the limitations imposed on the turbulence model by the Boussinesq approximation, which concentrates the energy of the eddies in the vortex shedding frequency, to generate stronger vortices and therefore prevent the transfer of energy towards smaller scales of turbulence. Moreover, two-equation turbulence models tend to 


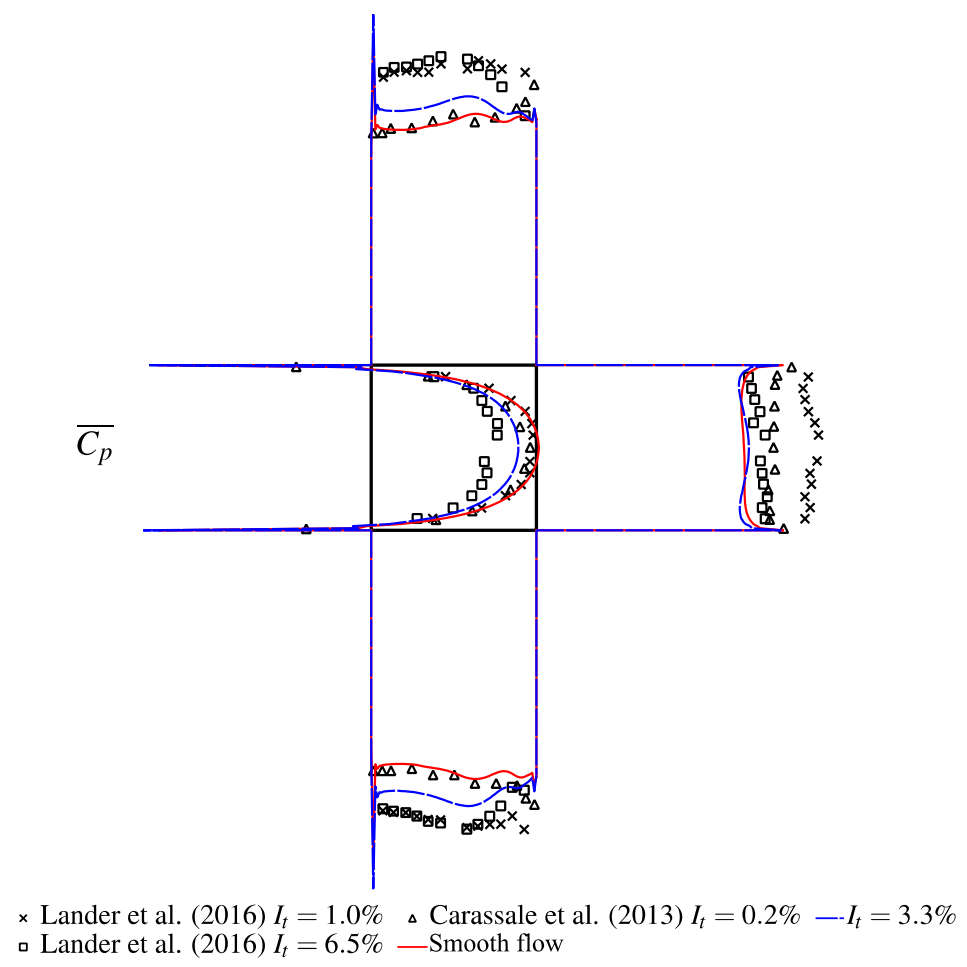

(a)

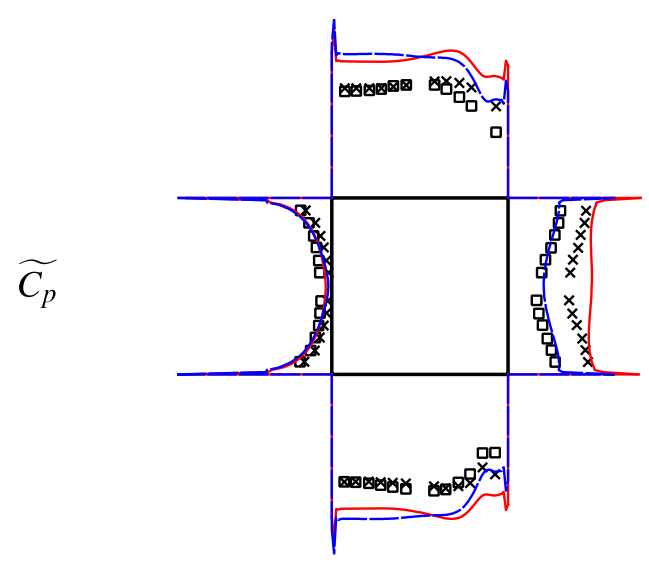

$\times$ Lander et al. (2016) $I_{t}=1.0 \%$ - Smooth flow

口 Lander et al. (2016) $I_{t}=6.5 \%-I_{t}=3.3 \%$

(b)

Figure 5: Pressure coefficient distributions for the two levels of turbulence intensity considered. (a) Mean pressure coefficient distribution. Negative values points outward the section and the side of the square cylinder is equal to a value of unity; and (b) Fluctuating component of the pressure coefficient distribution. Positive values points outward the section and the side of the square cylinder is equal to a value of unity. 


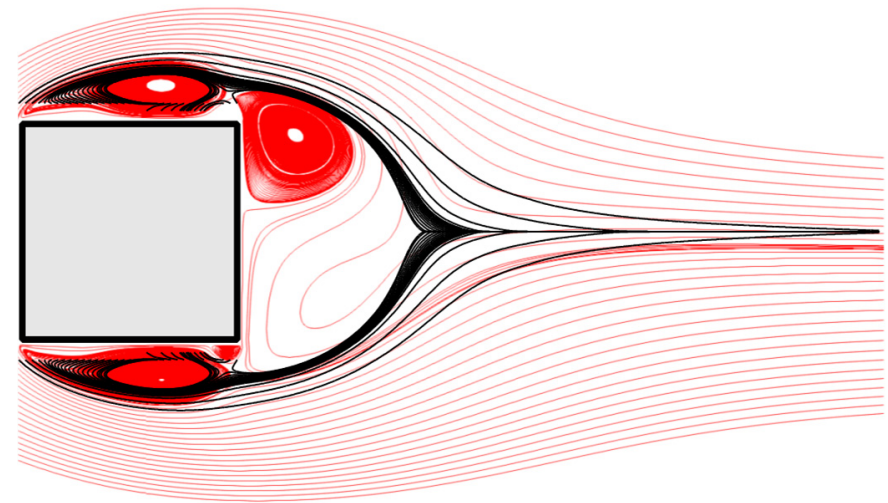

(a)

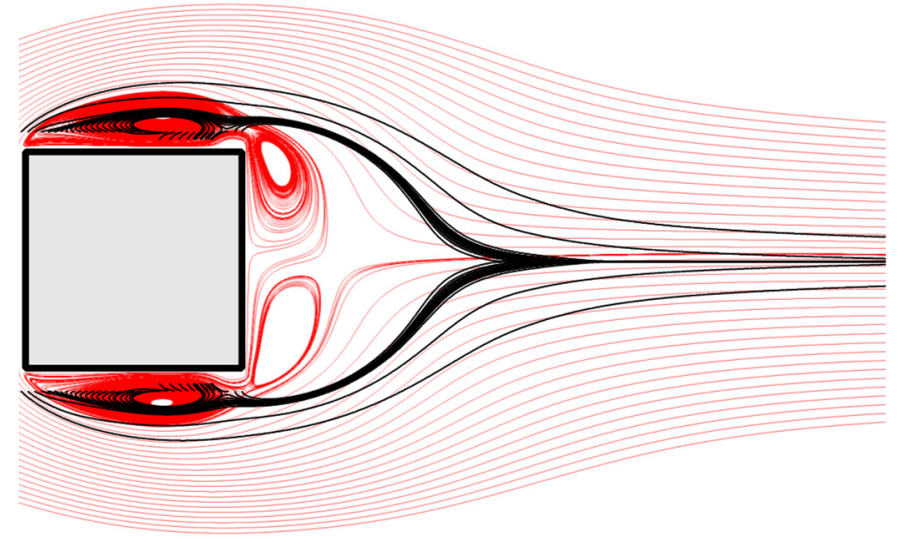

(b)

Figure 6: Time-averaged streamlines of the two cases with different turbulence intensity considered in the present study. The black lines correspond with the experimental data reported by Lander et al. [4] and the red lines are the ones corresponding to the present study. (a) Lander et al. 2016 It $=1.0 \%$ [4] vs present study smooth flow; and (b) Lander et al. 2016 It $=6.5 \%$ [4] vs present study $I t=3.3 \%$.

anticipate turbulent regimes at Reynolds numbers one order of magnitude lower than in experimental observations [15]. This prevents an accurate simulation of the transition phenomenon in the shear layer, which in this case could be further enhanced due to the addition of extra turbulent energy generated by the rod.

\section{CONCLUSIONS}

The main goal of this study has been to assess the feasibility of using relatively inexpensive numerical simulations that do not resolve the scales of turbulence to address the effects of free stream turbulence in bluff bodies. In the present study, 2D URANS simulations of a single rod, a single square and a combination of both have been carried out to study the effects of rod-generated small-scale turbulence on drag coefficient, Strouhal number, pressure coefficient distributions and time-averaged flow features. 
The reported results have proven the ability of 2D URANS simulations to reproduce the effects caused by rod-generated uniform small-scale turbulence in the pressure distributions and mean flow features.

The proposed approach will form a suit of further study by completing a more systematic study for different free stream turbulence levels. Future work to be undertaken by the authors will comprise the consideration of different angles of attack and fluid-structure interaction problems such as lock-in and galloping under turbulent flow.

\section{ACKNOWLEDGEMENTS}

This research has been funded by the Galician Regional Government under the Competitive Reference Research Groups Program under the reference code ED431C2017/72. The computations have been carried out in the computer cluster Breogán and in the Galician Supercomputing Centre (CESGA). The authors gratefully acknowledge the support received.

\section{REFERENCES}

[1] Gartshore, I.S., The effects of free stream turbulence on the drag of rectangular twodimensional prisms. Dept. BLWT-4-73, University of Western Ontario, Canada, 1973.

[2] Kwok, K.C.S. \& Melbourne, W.H., Freestream turbulence effects on galloping. ASCE Journal of the Engineering Mechanics Division, 106(2), pp. 273-288, 1980.

[3] Kwok, K.C.S., Turbulence effect on flow around circular cylinder. ASCE Journal of the Engineering Mechanics Division, 112(11), pp. 1181-1197, 1986.

[4] Lander, D.C., Letchford, C.W., Amitay, M. \& Kopp, G.A., Influence of the bluff body shear layers on the wake of a square prism in a turbulent flow. Physical Review Fluids, 1, article no. 044406, 2016.

[5] Patruno, L. \& Ricci, M., A systematic approach to the generation of synthetic turbulence using spectral methods. Computer Methods in Applied Mechanics and Engineering, 340, pp. 881-904, 2018.

[6] Lamberti, G., García-Sánchez, C., Sousa, J. \& Gorlé, C., Optimizing turbulent inflow conditions for large-eddy simulations of the atmospheric boundary layer. Journal of Wind Engineering and Industrial Aerodynamics, 177, pp. 32-44, 2018.

[7] García-Sánchez, C., Van Tendeloo, G. \& Gorlé, C., Quantifying inflow uncertainties in RANS simulations of urban pollutant dispersion. Atmospheric Environment, 161, pp. 263-273, 2017.

[8] Antoniou, N., Motazeri, H., Neophytou, M. \& Blocken, B., CFD simulation of urban microclimate: Validation using high-resolution field measurements. Science of the Total Environment, 695, article no. 133743, 2019.

[9] Ricci, A. \& Blocken, B., On the reliability of the 3D steady RANS approach in predicting microscale wind conditions in seaport areas: The case of the Ijmuiden sea lock. Journal of Wind Engineering and Industrial Aerodynamics, 207, article no. 104437, 2020.

[10] Wilcox, D.C., Turbulence modelling for CFD, DCW Industries: La Cañada, 2006.

[11] Menter, F. \& Esch, T., Elements of industrial heat transfer prediction. Proceedings of the 16th Brazilian Congress of Mechanical Engineering, 20, pp. 117-127, 2001.

[12] Simiu, E. \& Scanlan, R.H., Wind effects on structures, John Wiley \& Sons: New York, 1986.

[13] Vickery, B.J., Fluctuating lift and drag on a long cylinder of square cross-section in a smooth and in a turbulent stream. Journal of Fluid Mechanics, 25, pp. 481-494, 1966. 
114 Advances in Fluid Dynamics with emphasis on Multiphase and Complex Flow

[14] Carassale, L., Freda, A. \& Marrè-Brunenghi, M., Experimental investigation on the aerodynamic behavior of square cylinders with rounded corners. Journal of Fluids and Structures, 44, pp. 195-204, 2014.

[15] Collie, S., Gerritsen, M. \& Jackson, P., Performance of two-equation turbulence models for flat plate flows with leading edge bubbles. Journal of Fluids and Structures, 130, pp. 021201-1-11, 2008.

[16] Townsend, A., Momentum and energy diffusion in the turbulent wake of a cylinder. Proceedings of the Royal Society A, 197, pp. 124-140, 1949. 